\title{
On the use of Implication Intensity for matching ontologies and textual taxonomies
}

\author{
Jérôme David, Fabrice Guillet, Henri Briand, and Régis Gras \\ Laboratoire d'Informatique de Nantes Atlantique \\ Equipe COnnaissances \& Décision \\ Site Ecole Polytechnique de l'Université de Nantes \\ La Chantrerie - BP 50609 - 44306 Nantes cedex 3 \\ \{jerome.david,fabrice.guillet, henri.briand\}@univ-nantes.fr, \\ regisgra@club-internet.fr
}

Summary. At the intersection of data mining and knowledge management, we shall hereafter present an extensional and asymmetric matching approach designed to find semantic relations (equivalence and subsumption) between two textual taxonomies or ontologies. This approach relies on the idea that an entity $A$ will be more specific than or equivalent to an entity $B$ if the vocabulary (i.e. terms and data) used to describe $A$ and its instances tends to be included in that of $B$ and its instances. In order to evaluate such implicative tendencies, this approach makes use of association rule model and Interestingness Measures (IMs) developed in this context. More precisely, we focus on experimental evaluations of IMs for matching ontologies. A set of IMs has been selected according to criteria related to measure properties and semantics. We have performed two experiments on a benchmark composed of two textual taxonomies and a set of reference matching relations between the concepts of the two structures. The first test concerns a comparison of matching accuracy with each of the selected measures. In the second experiment, we compare how each IM evaluates reference relations by studying their value distributions. Results show that the implication intensity delivers the best results.

Key words: Ontology alignment, ontology matching, association rule, interestingness measure

\section{Introduction}

In our information society, large databases and data warehouses have become widespread. This huge amount of information has led to the increasing demand for mining techniques for discovering knowledge nuggets. To meet this demand, the Knowledge Discovery in Databases (KDD) [16] community proposed the association rule model [1].

Initially motivated by the analysis of market basket data, the task of association rule mining aims at finding relations between items in datasets 
[9]. Association rules are propositions of the form "If antecedent then consequent", noted antecedent $\rightarrow$ consequent, representing implicative tendencies between conjunctions of valued attributes or items. Association rules have the advantage of being an easy and meaningful model for representing explicit knowledge. Furthermore, this unsupervised learning technique does not need particular information about knowledge to be discovered contrary to classical supervised techniques (such as decision trees). These advantages have motivated a great deal of research and the publication of association rule extraction algorithms such as Apriori [1,2]. Nevertheless, if only minimal support and confidence values are used, these algorithms typically produce many rules and it is hard to only select those which may interest the user. One way to face this problem is to use Interestingness Measures (IMs). IMs aim at assessing the implicative quality of association rules but also some useful characteristics such as novelty, significance, unexpectedness, nontriviality, and actionability $[9,19]$. IMs allow to rank and reduce the amount of rules, and consequently to help the user to choose the best ones according to his/her preferences.

The information society has also led to the development of the Web and then a great increase in the available data and information. In this vast Web, resources, often in textual form, tend to be organised into hierarchical structures. This hierarchical structuring of web contents ranges from large web directories (e.g. Yahoo.com, OpenDirectory) to online shop catalogs (e.g. Amazon.com, Alapage.com). Furthermore, with the arrival of the Semantic Web, such a hierarchical organisation is also used through OWL ontologies which aim at providing formal semantics of the web contents. Even if the use of hierarchies helps to structure web information and knowledge, the Web remains heterogeneous. Data exchanges and communications between software programs or software agents using hierarchical-organised data is consequently difficult. In order to address such interoperability problems, one must be able to compare such data structures and find matches between them. Thus, many matching methods have been proposed in the literature [27, 34, 35]. These methods aim at finding semantic relations (i.e. equivalence, subsumption, etc) between entities (i.e. directories, categories, concepts, properties) defined in different hierarchical structures (filesystems, schemas, ontologies). Even if the proposed approaches are issued from different communities, they mostly use similarity measures and as a consequence, a majority of them are restricted to finding equivalence relations only.

At the intersection of these two research fields, we proposed to use the association rule paradigm for matching ontological structures [11]. Our original approach, named AROMA (Association Rule Ontology Matching Approach), heavily relies on the asymmetric nature of association rules, which allows it to match not only equivalence relations but also subsumption relations between entities. The consideration of subsumption between entities helps to characterise more precisely the matching relations between hierarchical structures regarding only similarity based approaches. Also, it allows enhancements the output matches. Furthermore, unlike most approaches designed for matching 
schema or ontologies, AROMA relies heavily on extensional data provided with structures. This type of matcher, named an instance-level matcher [34], is especially designed to work on structures with limited schema information (i.e. only concept or element names and a partial order relation between them). For example, AROMA can deal with textual hierarchies, such as Web directories or semi-structured data.

The main objective of this chapter is to show and explain the relevance of the Implication Intensity measure relative to other IMs in the context of the problem of matching text hierarchies.

This chapter is organised as follows: in a first section, we present related work concerning ontology/schema matching and propose a classification. Then, we focus on IMs proposed in the literature. First, we classify measures according to three criteria. Then, we use this classification to justify the selection of the best IMs according to our matching context. In the second section, we detail the two stages of AROMA methodology and, we describe a criterion for reducing rule redundancy and enhancing the accuracy of matching results. The last section reports the results of two experiments made on a well-known benchmark provided with two catalogs and a set of reference matching relations. The first experiment evaluates the selected IMs according to a classical information retrieval accuracy measure: the F-measure. In the second experiment, we compare the value distributions of IMs obtained on relevant and non-relevant relation sets.

\section{Related work}

\subsection{Textual taxonomy matching}

For the last six years, ontology and schema matching have been widely studied and many approaches have been proposed in the literature. These methods come from different communities such as artificial intelligence [18, 20, 7], databases [29, 12, 32], graph matching [30, 23], information retrieval [28], machine learning [13, 31, 36], natural language processing and statistics [24]. Although they are heterogeneous and consequently difficult to compare, preliminary afforts have been result in surveys of matching techniques [27, 34, 35].

One survey [34] focuses on database schema matching techniques and proposes a classification which discriminates the extensional or element-based approaches from the intensional or only-schema-based approaches. While many efforts are concentrated around the intensional matchers, few extensional approaches have been proposed in the literature.

A survey of intensional matchers can be found in [35]. The authors propose two classifications of this type of matchers. The first one permits us to distinguish methods according to their granularity and their interpretation of the input information (it distinguishes element-level and structure-level techniques and then the syntactic, external and semantic methods). The second classification distinguishes three classes: 
1. the terminological approaches $(\mathrm{T})$ based on string similarity measures (TS) or based on measures which use terminological resources (TL) like WordNet;

2. the structural approaches (S) which compare two concepts from their internal structure (SI) (shared attributes or properties) or from their external structure (SE), that is to say their respective position within their taxonomy;

3. the semantic approaches (SEM), which use formal semantic model.

To sum up, most approaches use terminological matchers with stringsimilarities (Anchor-PROMPT [32], Coma [12], CMS [26], Cupid [29], SMATCH [20]) or/and external oracles such as Wordnet ([20], H-match [8]). They can also use structural matchers (Similarity Flooding [30], [8], GMO [23], Omap [36], [12], [18], [32], [29]). Some methods such as OLA [15], combine the various terminological and structural criteria. Only a few approaches use formal semantic models ([20], [18]).

\section{Extensional matchers}

In this section, we focus on extensional matchers since the methods designed for matching textual taxonomies (i.e. web directories, catalogs, etc.) heavily rely on textual content. Beacause, such data structures typically have poor schema information, extensional matchers are relevant. We briefly present four approaches which are explicitly designed to work on such data structures. Then, we propose a synthetic classification of these approaches based on their differences.

GLUE [13]. This conceptual hierarchy matching tool uses machine learning techniques. It combines two strategies: the estimation of joint probabilities between concepts and the relation labeller. In the first strategy, for the joint probability estimation, the two hierarchies must share the same set of textual documents. As it is often not the case, the authors propose to classify the documents associated to the concepts of the first hierarchy to the concepts of the second one. This classification process uses several machine learning classifiers: a naive Bayesian classifier on the textual content of the documents, and a naive Bayesian classifier on the names of concepts concatenated with the names of ascendant concepts (concept path). These two classifiers are firstly trained on the documents associated with each hierarchy. The different classifier predictions are then combined by using the meta-learner. The joint probability is evaluated using Jaccard similarity.

oPLMap [31]. This tool is based on a logical and probabilistic model: DATALOG. It aims at finding the matching set which maximises the alignment probability. This method considers rules of the form $S_{i} \rightarrow T_{j}$ and evaluates the confidence of the rules by combining several classifiers. It relies on terminological classifiers for concept names (concept name identity, Jaccard measure on words composing concept names, Jaccard measure by considering 
the concept path name) and machine learning classifiers for textual documents (kNN classifier and Naive Bayes text classifier on the documents). The oPLMap approach also uses some constraints for taking into account the structure of the taxonomies. In the output, this method provides a set of n-to-n mapping elements valued by a probability measure.

Hical or SBI [25]. This method uses a statistical test, named $\kappa$-statistic [10], on shared documents for determining matching. $\kappa$-statistic tests the null hypothesis: " $\kappa=0$ ". A relation between concepts holds if the null hypothesis can be dissmissed with a significance level of $5 \%$. Hical proposes a top-down approach in order to reduce the computing time.

CAIMAN matching service [28]. This matching method is enclosed in the CAIMAN system for facilitating the exchange of relevant documents between geographically dispersed people within their communities of interest. In the CAIMAN matching service, each document is represented by a document vector composed of words and word frequencies weighted by the TF/IDF measure. Then, the characteristic vectors of concepts are computed from the document vectors by using the Rocchio classifier. Finally, similarities between concepts are deduced by evaluating the cosine value between characteristic vectors of concepts. The output matching is a one-to-one matching: for each source concept, the method retains only the target concept for which the measure is maximised.

\section{Classification of textual taxonomy matchers}

\begin{tabular}{|l|l|l|l|l|}
\hline & $\begin{array}{l}\text { Kind of rela- } \\
\text { tions }\end{array}$ & Measure & $\begin{array}{l}\text { Comparison } \\
\text { level }\end{array}$ & $\begin{array}{l}\text { Pre- } \\
\text { processing } \\
\text { step }\end{array}$ \\
\hline GLUE & equivalence & Jaccard & document level & $\begin{array}{l}\text { classification of } \\
\text { documents }\end{array}$ \\
\hline oPLMap & equivalence & $\begin{array}{l}\text { confidence (con- } \\
\text { ditional proba- } \\
\text { bility) }\end{array}$ & document level & $\begin{array}{l}\text { classification of } \\
\text { documents }\end{array}$ \\
\hline Hichal & equivalence & k-statistic & document level & none \\
\hline CAIMAN & equivalence & cosines & term level & $\begin{array}{l}\text { characteristic } \\
\text { vectors compu- } \\
\text { tation }\end{array}$ \\
\hline AROMA & $\begin{array}{l}\text { subsumption, } \\
\text { equivalence }\end{array}$ & $\begin{array}{l}\text { Implication In- } \\
\text { tensity term level }\end{array}$ & $\begin{array}{l}\text { selection } \\
\text { sets of relevant } \\
\text { terms }\end{array}$ \\
\hline
\end{tabular}

Table 1. Comparison of hierarchy matchers 
Table 1 compares of four matchers and AROMA method discussed in Section 3. We analyse them according to four criteria:

- The kind of relation that the method considers. It can be equivalence or subsumption relations.

- The measure used by the method for evaluating a potential matching between concepts. The measures used in the five approaches are Jaccard similarity, the conditional probability (called confidence in the association rule community), $\kappa$-statistic, cosine and the Implication Intensity.

- The comparison level shows how concepts are represented and then explains on which basis the similarity or measure is calculated. We denote two modalities: (1) the document level for methods comparing shared documents between entities; and (2) the term level for methods which represent entities by a set of terms.

- The kind of pre-processing step refers to the kind of preprocessing applied to the extension in order to be able to calculate similarities. This characteristic is partially determinated by the type of comparison level. In the case of document level approaches, documents can be classified from each structure into the other one so that they share the same set of documents. Term level approaches use linguistic processing in order to extract terms and then select and/or weight these terms according to their relevance to the studied entities.

We can see that only AROMA considers the subsumption relation. The others are restricted to the equivalence. Concerning the measures used, three methods use similarities (which are symmetric) while only oPLMap and AROMA use asymmetric measures. Nevertheless, oPLMap does not seem to use its asymmetric measure to find subsumption relations between concepts. We can also note that Hichal and AROMA use measures based on a statistical model. For the comparison level, three methods use the documents for representing the extension of concepts from which they calculate the measure values. Only CAIMAN and AROMA work at the terminological level by representing concepts by sets of terms (characteristic vectors or relevant term sets). Finally, in order to work on a common document base, the GLUE and oPLMap methods use a combination of classifiers for the documents.

\subsection{Interestingness Measures}

In the framework of association rule discovery, and in order to select the most interesting rules, many Interestingness Measures (IMs) have been proposed and studied (see [3] [22] [19] [37] for a survey). In this context, some researchers are interested in principles and properties defining a good IM ([33], [22], [37], $[17],[3])$, while others work on the comparison of IMs from a data-analysis point of view.

According to our objective of hierarchy matching, we selected some IMs that may be relevant for our work. In the context of AROMA, unlike associ- 
ation rule discovery, an IM is not used for ranking rules in a post-processing step, but during the rule extraction process. In order to be able to choose a threshold value more easily, we retained only IMs respecting the principle of minimal and maximal value [22]. IMs respecting this behaviour are more intelligible to a user.

In this section, we firstly introduce notations used for representing association rules and their characteristics. Then, we classify selected IMs according to three main criteria proposed in $[6,4]$. The resulting taxonomy of IMs shows their main properties and permits understanding of their behaviours and semantics.

\section{Definition of association rule and notations}

In this section we use the following notation: A finite set $T$ of $n$ individuals is described by a set $I$ of $p$ items. Each transaction $t$ can be considered as an itemset, so that $t \subseteq I$. $A=\{t \in T ; a \subseteq t\}$ is the extension of itemset $a$ and $\bar{B}=T-\left\{t^{\prime} \in T ; b \subseteq t^{\prime}\right\}$ is the extension of $\bar{b}$. An association rule [1] is an implication of the form $a \rightarrow b$, where $a$ and $b$ are disjoint itemsets. In practice, it is quite common to observe some transactions which contain $a$ and not $b$ without in spite of a general trend to have $b$ when $a$ is present. Then, we introduce the quantities $n_{a}=\operatorname{card}(A), n_{\bar{b}}=\operatorname{card}(\bar{B})$ and $n_{a \wedge \bar{b}}=\operatorname{card}(A \cap \bar{B})$.

\section{Taxonomy of IMs}

The taxonomy classifies IMs according to three criteria. The first one concerns subject of IMs (deviation from independence or equilibrium) and the second one, the nature of IMs (descriptive or statistical). The last one, the scope of the IMs (quasi-implication, quasi-conjunction, quasi-equivalence), explains the semantics of the measure according to logical operators.

- Subject. A family of IMs evaluate the deviation from the independence situation where the number of counter-examples is equal to those expected in a random case $\left(n_{a \bar{b}}=n_{a} \cdot n_{\bar{b}} / n\right)$. These measures have a fixed value at the independence. The other family of IMs evaluate the deviation from the equilibrium. The equilibrium situation is reached when the number of counter-examples and examples are equal in number $\left(n_{a \bar{b}}=n_{a b}\right)$.

- Nature. The nature of an IM can be descriptive or statistical. The descriptive measures are not influenced by a proportional expansion of the cardinalities taken into account. A descriptive IM $m$ satisfies $m\left(n_{a}, n_{b}, n_{a \bar{b}}, n\right)=$ $m\left(\alpha . n_{a}, \alpha . n_{b}, \alpha . n_{a \bar{b}}, \alpha . n\right)$ with $\alpha .>0$. Conversely, the statistical measures vary with the expansion of cardinalities. According to the authors, this type of IM allows the validity of rules to be statistically assessed. Some of these measures are also particularly effective at detecting rules that have novel consequents, i.e. consequents that have not been seen in previous rules. For example, Implication Intensity decreases with an increase 
of $n_{b}$, and thus gives preference to statistically valid rules having novel consequents.

- Scope. Finally, this last way of distinguishing IMs relies on the idea that IMs may evaluate a proximity between the rule and a logical configuration such as an implication, a conjunction, or an equivalence [4]. To qualify the scope of an IM, we will use the terms quasi-implication, quasi-conjunction and quasi-equivalence indexes because rules are not strict logical propositions since they may have counter-examples. Furthermore, some IMs only evaluate the tendency to verify the consequent when the antecedent is true (i.e. they only consider the examples of a rule). Such measures (e.g. the Confidence or IPEE [5] measures) are not classified in the Table 2.

For each modality of the scope characteristic of an IM, Table 2 shows their symbol, counter-examples, equivalent linkage and property that the IM must respect. By comparing this table and the semantic relations defined by [20], we can see that based on their scope, measures could be more or less adapted for mining certain types of semantic relations. Subsumption relations must be evaluated by quasi-implication measures, overlapping relations by quasi-conjunction measures and equivalence relations by quasi-equivalence measures. In schema or ontology matching, methods often rely on quasiconjunction measures for evaluating equivalence relations. of the approaches considered in Section 2.1, only Hichal uses an index ( $\kappa$ or kappa) that is a quasi-equivalence measure. GLUE uses a quasi-conjunction index (Jaccard) and oPLMap uses a rule index (confidence or conditional probability).

\begin{tabular}{|l|c|c|c|}
\hline & quasi-implication & quasi-conjunction & quasi-equivalence \\
\hline Symbol & $\Rightarrow$ & $\leftrightarrow$ & $\Leftrightarrow$ \\
\hline Counter-examples & $a \wedge \bar{b}$ & $a \wedge \bar{b}$ & $a \wedge \bar{b}$ \\
& & $\bar{a} \wedge b$ & $\bar{a} \wedge b$ \\
& & $\bar{a} \wedge \bar{b}$ & \\
\hline equivalence & $a \supset b \equiv \bar{b} \supset \bar{a}$ & $a \leftrightarrow b \equiv b \leftrightarrow a$ & $a \leftrightarrow b \equiv \bar{a} \leftrightarrow \bar{b}$ \\
\hline IM's property & $I(a \rightarrow b)=I(\bar{b} \rightarrow \bar{a})$ & $I(a \rightarrow b)=I(b \rightarrow a)$ & $I(a \rightarrow b)=I(\bar{a} \rightarrow \bar{b})$ \\
\hline
\end{tabular}

Table 2. Scope of IMs

Table 3 shows, for each selected IM, its scope (rule $(\rightarrow$ ), quasi-implication $(\Leftrightarrow)$, quasi-conjunction $(\leftrightarrow)$, quasi-equivalence $(\Leftrightarrow)$ ), its nature (statistical (S) or descriptive (D)), its subject (deviation from independence (I) or equilibrium (E)), its fixed value in the independence or equilibrium situation (depending on its subject) and its formula. 


\begin{tabular}{|l|l|l|l|l|c|}
\hline Measure & Scope & Nature & Subject & Fixed value & Formula \\
\hline II & $\Rightarrow$ & S & I & 0.5 & $P\left(n_{a \bar{b}}<{\left.\operatorname{Poisson}\left(\frac{n_{a} \cdot n_{\bar{b}}}{n}\right)\right)}_{1}\right.$ \\
\hline Loevinger & $\Rightarrow$ & D & I & 0 & $1-\frac{n_{a \cdot n} \bar{b}}{n_{a} \cdot n_{\bar{b}}}$ \\
\hline IPEE & $\rightarrow$ & S & E & 0.5 & $P\left(n_{a \bar{b}}<\operatorname{Binomial~}\left(n_{a}, 1 / 2\right)\right)$ \\
\hline Confidence & $\rightarrow$ & D & E & 0.5 & $n_{a b} / n_{b}$ \\
\hline $\begin{array}{l}\text { Likelihood } \\
\text { Linkage } \\
\text { Analysis }\end{array}$ & $\leftrightarrow$ & S & I & 0.5 & $P\left(n_{a b} \geq{\left.\operatorname{Poisson}\left(\frac{n_{a} \cdot n_{b}}{n}\right)\right)}\right.$ \\
\hline
\end{tabular}

Table 3. Selected IMs and their properties

\section{AROMA methodology}

AROMA (Figure 1) was designed to find matching between conceptual hierarchies populated from textual documents. This method permits the discovery of a set of significant association rules holding between concepts obtained from two hierarchical structures and evaluated by the Implication Intensity measure. AROMA takes, as input, two conceptual hierarchies $\mathcal{H}_{1}$ and $\mathcal{H}_{2}$, each defined as a tuple $\mathcal{H}=(C, \leq, D, \sigma)$, where $C$ is the set of concepts, $\leq$ is the partial order organising concepts into a taxonomy, $D$ is the set of textual documents, and $\sigma$ is the relation associating a set of documents to each concept (i.e. for a concept $c \in C, \sigma(c)$ represents the documents associated to $c$ ). Thanks to the first part of the method concerning the acquisition and the selection of relevant terms for each concept, we are able to redefine each hierarchy as a tuple $\mathcal{H}^{\prime}=(C, \leq, T, \gamma)$ where $T$ is the set of relevant terms selected. In order to consider the partial order, we assume that a term associated with a concept is also associated with its parent concepts, and thus, we extend $\gamma$ to the relation $\gamma^{\prime}$ as follows: $\gamma^{\prime}(c)=\bigcup_{c^{\prime} \leq c} \gamma\left(c^{\prime}\right)$.

\subsection{Association rules discovery between hierarchies}

The second stage of AROMA consists of the discovery of implicative matching relations between concepts by evaluating association rule between their respective sets of relevant terms. The algorithm takes in two pre-processed hierarchies $\mathcal{H}^{\prime}{ }_{1}$ and $\mathcal{H}^{\prime}{ }_{2}$ and considers only the terms shared by the two structures. The set of common terms for the two hierarchies $\mathcal{H}^{\prime}{ }_{1}$ and $\mathcal{H}^{\prime}{ }_{2}$ is noted $T_{1 \cap 2}=T_{1} \cap T_{2}$. The relation $\gamma_{1 \cap 2}^{\prime}$ associates a subset of $T_{1 \cap 2}$ for each concept $c \in C_{1} \cup C_{2}$ :

$$
\gamma_{1 \cap 2}^{\prime}(c)=\left\{\begin{array}{l}
\gamma_{1}^{\prime}(c) \cap T_{2} \text { if } c \in C_{1} \\
\gamma_{2}^{\prime}(c) \cap T_{1} \text { if } c \in C_{2}
\end{array}\right.
$$

The extracted rules are of the form $a \rightarrow b$ and are valued by a $\varphi(a \rightarrow b)$ value. A valid rule $a \rightarrow b$ (i.e. rule having a $\varphi(a \rightarrow b)$ value greater than or equals to a chosen threshold) represents a quasi-implication (i.e. an implication allowing some counter-examples) from the set of relevant terms of the concept 


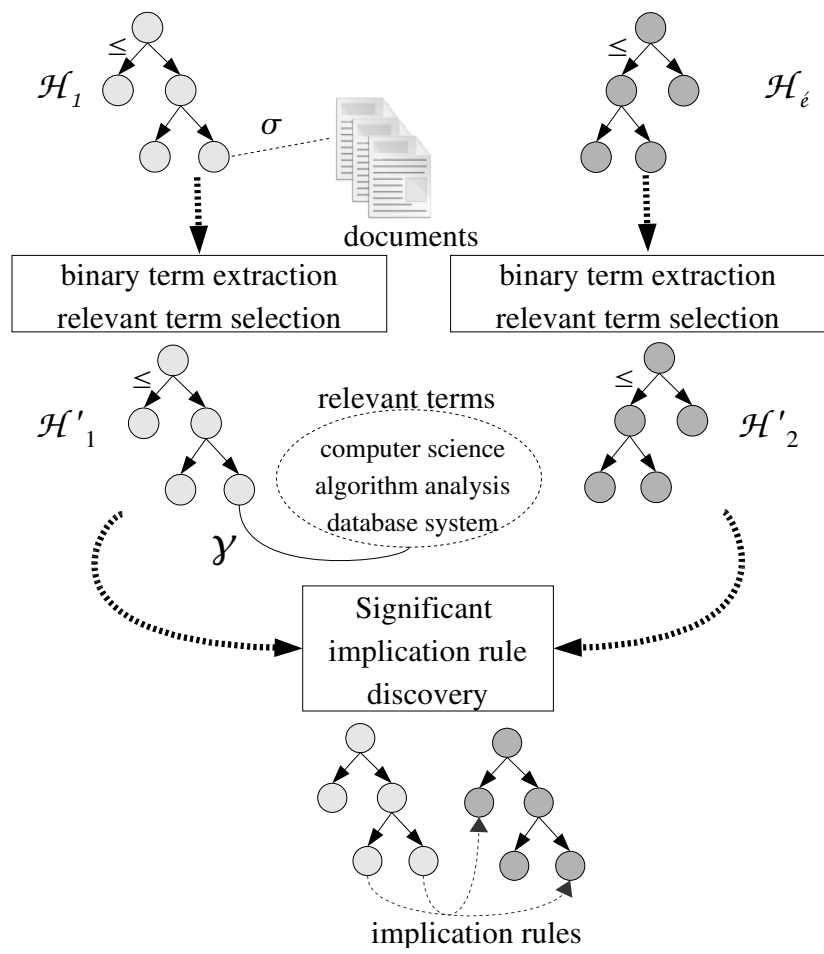

Fig. 1. The AROMA approach

$a$ into the set of relevant terms of the concept $b$. The existence of such a valid rule means that the concept $a$ (issued from $\mathcal{H}_{1}$ ) is probably more specific than or equivalent to the concept $b$ (issued from $\mathcal{H}_{2}$ ).

\subsection{Selection of significant rules}

The algorithm provides a top-down search of association rules. It uses two criteria for selecting significant rules and reduce redundancy. Then, a rule $a \rightarrow b$ (between the concepts $a \in C_{1}$ and $b \in C_{2}$ ) will be significant if it respects the two following criteria:

$$
\begin{gathered}
\varphi(a \rightarrow b) \geq \varphi_{r} \\
\forall x \geq a, \forall y \leq b, \varphi(x \rightarrow y) \leq \varphi(a \rightarrow b)
\end{gathered}
$$

The first criterion (Equation 2) guarantees the quality of the implication tendency between the two concepts for a given threshold $\varphi_{r}$. The Implication Intensity of the rule $a \rightarrow b$ is explained in Figure 2 and defined as follows:

$$
\varphi(a \rightarrow b)=1-\operatorname{Pr}\left(N_{a \wedge \bar{b}} \leq n_{a \wedge \bar{b}}\right)
$$




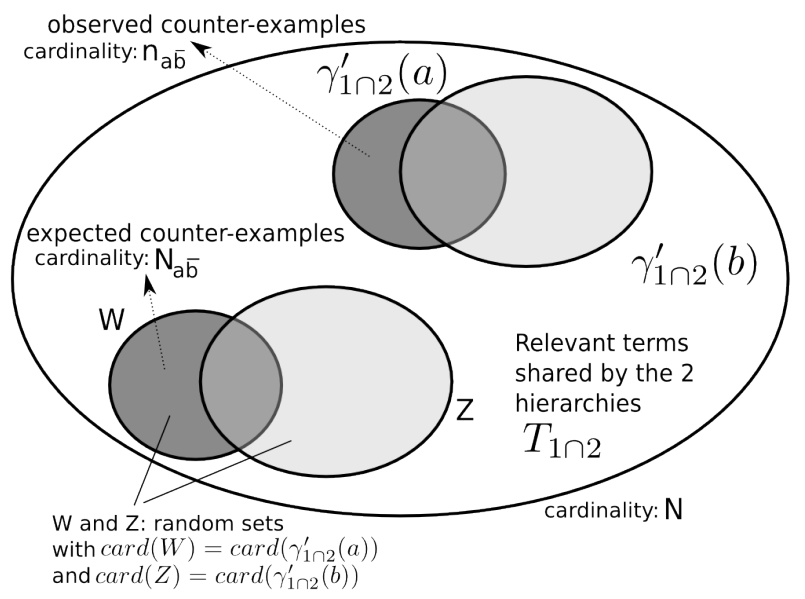

Fig. 2. Implication Intensity of a rule $a \rightarrow b$

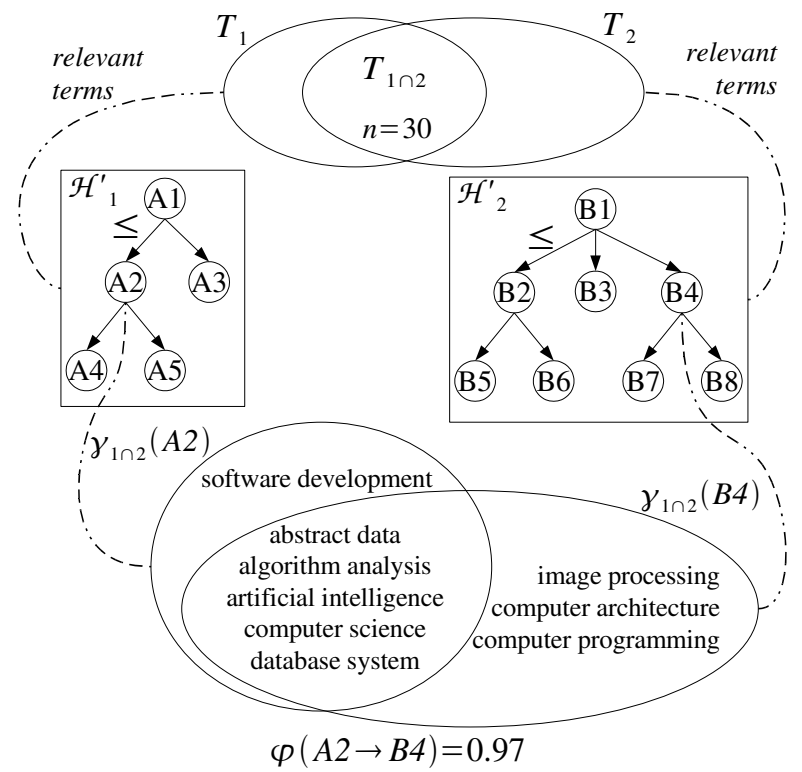

Fig. 3. Evaluation of rules 
where $n_{a \wedge \bar{b}}=\operatorname{card}\left(\gamma_{1 \cap 2}(a)-\gamma_{1 \cap 2}(b)\right)$ is the number of relevant terms for concept $a$ that are not relevant for concept $b . N_{a \wedge \bar{b}}$ is the random number of relevant terms for concept $a$ that are not relevant for concept $b$.

For example (Figure 3), the rule $A 2 \rightarrow B 4$ has $n_{A 2 \wedge \overline{B 4}}=1$ counterexamples. Its Implication Intensity value is calculated using a Poisson law (which is a possible model for the Implication Intensity [21]):

$$
\varphi(A 2 \rightarrow B 4)=\sum_{k=0}^{n_{A 2 \wedge \overline{B 4}}} e^{-\lambda} \cdot \frac{\lambda^{k}}{k !}=0,97
$$

where $\lambda=n_{A 2} . n_{\overline{B 4}} / n=6 .(30-8) / 30$.

The second criterion (Equation 3 ) verifies the generativity of the rule and thus allows the redundancy to be reduced in the extracted rules set. Indeeds, a valid rule (i.e. a rule satisfying the first criterion) is significant if there does not exist a more generative rule having an Implication Intensity value greater than or equals to it. A rule $x \rightarrow y$ is more generative than a rule $u \rightarrow v$ if $u \leq x$ and $y \leq v$ (with $x \rightarrow y \neq u \rightarrow v$ ). For example (Figure 3), the rules $A 2 \rightarrow B 7, A 2 \rightarrow B 8, A 1 \rightarrow B 4, A 1 \rightarrow B 7$, and $A 1 \rightarrow B 8$ are more generative than the studied rule $A 2 \rightarrow B 4$. The rule $A 2 \rightarrow B 4$ will be significant and thus selected if none of its generative rules have a $\varphi$ value greater than or equals to its $\varphi$ value.

\section{Experimental results}

The experiments presented in this section concern only the second part of the AROMA (i.e. the rule selection phase). After describing the data used for the experiments, we first compare the performance of the measures in terms of the F-Measure, which aggregates precision and recall. Next, we describe an analysis of the distribution of the measure values on two sets of matching relations: a set of hand-made reference matching relations, noted $R+$ and a set of irrelevant relations $R-$.

\subsection{Analysed data}

The experiments used the "Course catalog" benchmark [14]. This benchmark is composed of two catalogs of courses descriptions which are offered at the Cornell and Washington universities. The courses descriptions are hierarchically organised into schools and colleges and then into departments and centers within each college. These two hierarchies contain respectively 166 and 176 concepts to which are associated 4360 and 6957 textual course descriptions. The benchmark data also include a set of 54 manually matched relations from concepts of the Cornell catalog to the Washington catalog. Only equivalence relations are included in the manually matched set. 


\subsection{Evaluation of IMs}

Here, we describe the evaluation performed by the AROMA algorithm with each selected IM. For each measure, we varied the rule selection threshold $\varphi_{r}$ from 0 to 1 with a step of 1 percent. For each threshold value, the rule selection algorithms was executed twice, once to select implications from the Cornell concepts to the Washington concepts and the second time to select implications from the Washington concepts to the Cornell concepts. From the two implicative matching sets, we retained only equivalence relations by following this rule: if $A \rightarrow B$ and $B \rightarrow A$, then $A \leftrightarrow B$.

In order to evaluate the relevance of results according to the reference set $R+$, we use two standard metrics from information retrieval: the precision and the recall. These metrics are defined as follows: let $F$ be the set of matching pairs found using AROMA and $R+$ be the set of "reference" matching pairs. The precision $($ precision $=\operatorname{card}(F \cap R+) / \operatorname{card}(F))$ measures the ratio of the number of good matching pairs (i.e. matching pairs that are both in our result set and in the reference matching set) over the number of matching pairs found by AROMA. The recall (recall= $\operatorname{card}(F \cap R+) / \operatorname{card}(R+))$ measures the ratio of good matching pairs over the number of reference matching pairs. Finally, these two measures are aggregated into the F-measure (Dice's similarity between the sets $F$ and $R$ ) : $F-$ measure $=2$.precision .recall $/$ precision + recall .

Figure 4 shows a high correlation between Confidence and Loevinger in terms of efficiency. Their best F-measure scores (around 0.45) are obtained around a threshold of 0.3 . This threshold value is greater than those of independence situation for Loevinger but it is less than those of the equilibrium situation for Confidence. The IPEE index has tendency to have the same trend as Confidence and Loevinger. It has lower maximum F-measure values but it is more robust to the increase of the selection threshold value. Likelihood Linkage Analysis (LLA) don't have good F-measure scores. It obtains nearly constant recall values just above 0.5 but it has very bad precision values. Possibly the symmetric nature of this index is not well adapted to our algorithm. Finally, the best F-measure scores are obtained by the Implication Intensity index. Its best value is a little greater than 0.5 for a rule selection threshold fixed at 0.9 . We can also notice a stagnation of F-measure before the independence situation (rule selection threshold of 0.5 for II).

These results show that Implication Intensity is the most relevant measure in this context. The two descriptive indexes, Confidence and Loevinger, tend to have similar trends. The selected quasi-conjunction index, LLA, is not relevant for AROMA.

\subsection{Distributions of IMs}

In this experiment, we studied and compared how the IM's evaluated matching relations independently of the AROMA rule selection algorithms and their 


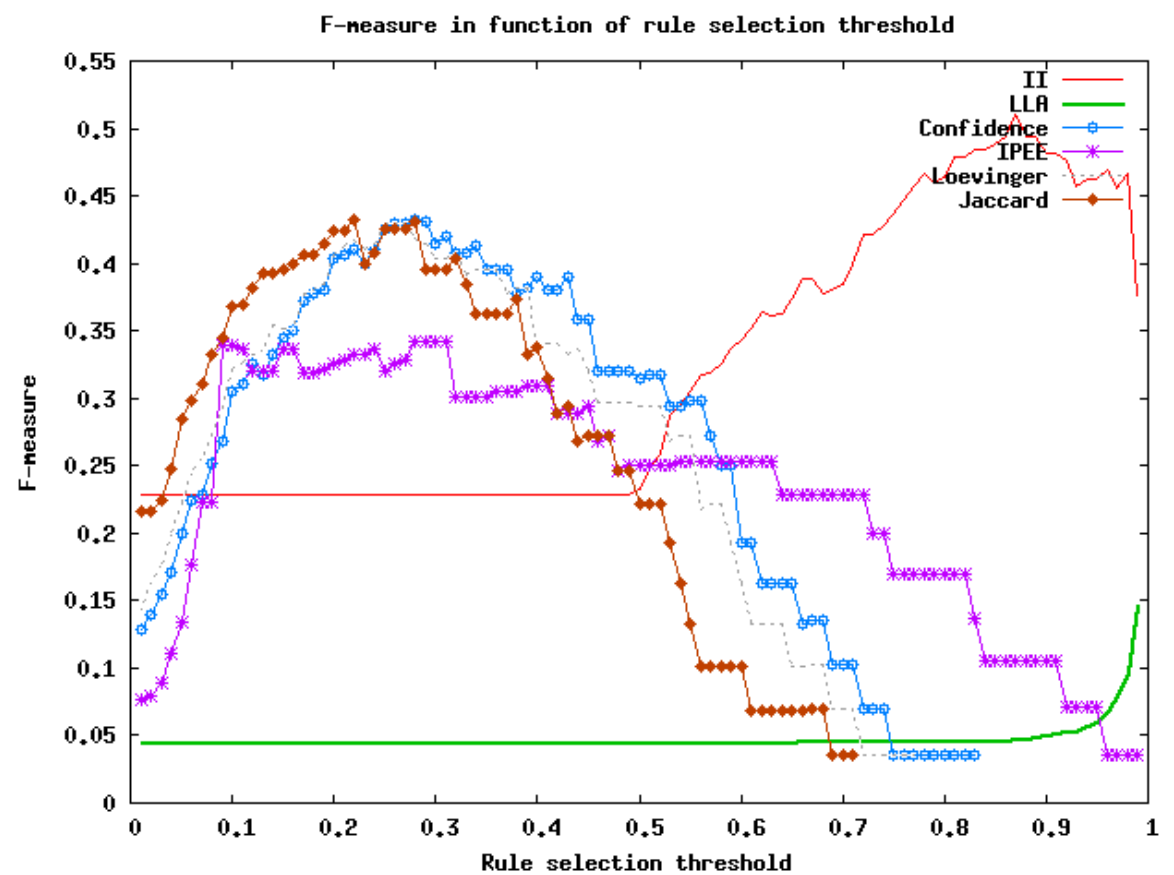

Fig. 4. Evolution of F-measure

selection criteria. Then, we propose to draw the values distributions of IM in two cases. The first case consisted of evaluating the relations from the manually matched set $R+$ and in the second case, we have tried the measures on the set of irrelevant relations $R-$. The set $R$ - was built manually and contains the same number of relations as the reference one $R+$. For these two evaluations, we performed the selection of relevant terms with the Implication Intensity measure for a threshold of 0.9 .

In the first test, for each manually matched relation represented by a pair $(A, B)$, we evaluated the rules $A \rightarrow B$ and $B \rightarrow A$. For each pair, we kept only the best value, in the cases where the studied measure was not symmetric.

Regarding Figure 5, the Confidence measure yields a value under 0.5 for the majority of the relations. According to the equilibrium situation, these relations are not relevant because their antecedents is more concomitant with the negation of their consequents than with their consequents. The results obtained with IPEE confirm this situation since many rules have a quality value near 0 . Nevertheless, the results found with Loevinger show that matching relations are good regarding the independence case. The majority of rules have a Loevinger value greater than 0 (only 4 relations have smaller values). 

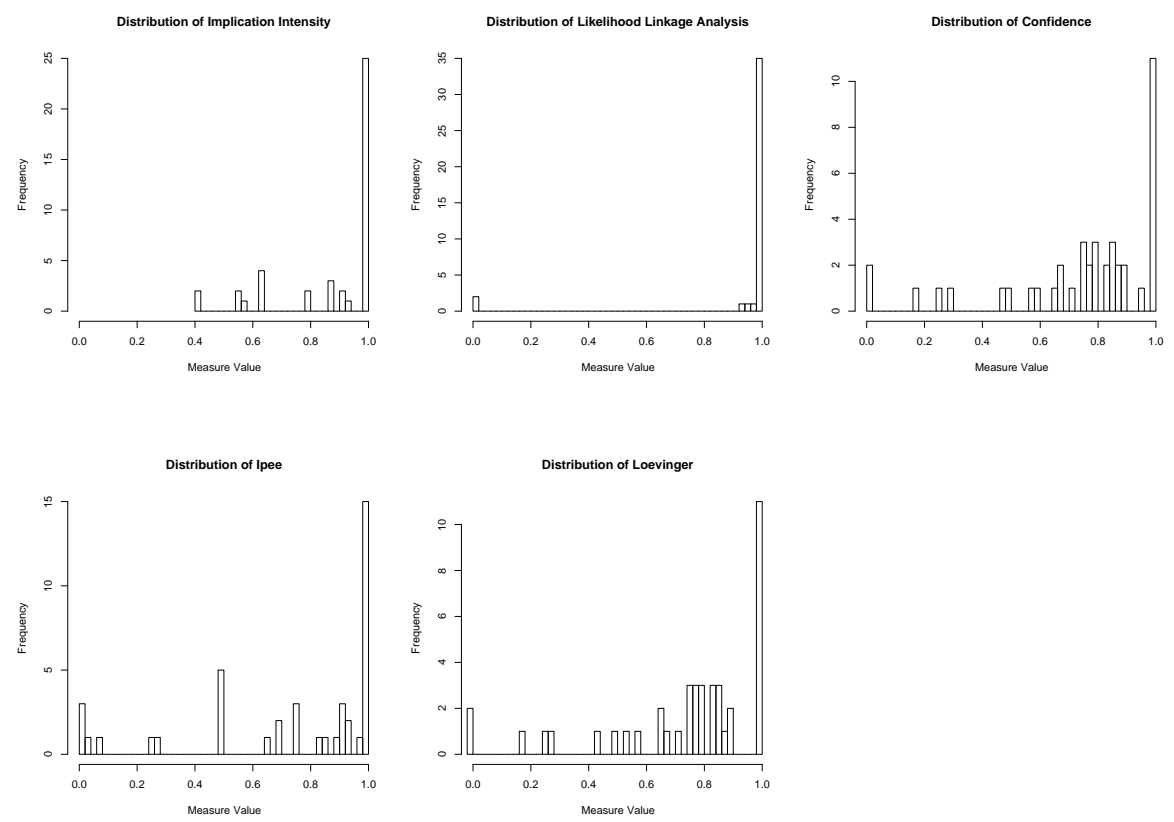

Fig. 5. Measures distributions on manual matching relations $R+$

Thus, we can say that their number of observed counter-examples is negatively correlated to the expected one under the independence hypothesis.

The two probabilistic measures of deviation from independence, the Implication Intensity (II) and the Likelihood Linkage Analysis measures, evaluate the majority of matching relations with good values. The second measure seems to work a little better on this benchmark. This result is not surprising because this second measure is symmetric and designed for evaluating quasiconjunction relations. But, unlike Implication Intensity, such a measure is not adequate in the case of mining quasi-implication relations between concepts.

Then, after the study of distribution of measures values on good matching relations, we performed a second test on a set of non-relevant relations. In this case, we consider the minimal value obtained for each pair of concepts. In Figure 6, all Confidence and IPEE values are near 0. Loevinger yields less than 0 for 30 rules, that is to say under the independence situation. II confirms this tendency because only 2 rules have a value greater than or equal to 0.5. Nevertheless, LLA evaluates a majority of rules with good values (i.e. greater than 0.5 obtained at the independence situation). Regarding Figures 5 and 6 , only Loevinger and II clearly distinguish the two sets of rules. These two sets of distributions show that IMs of deviation from independence work better 

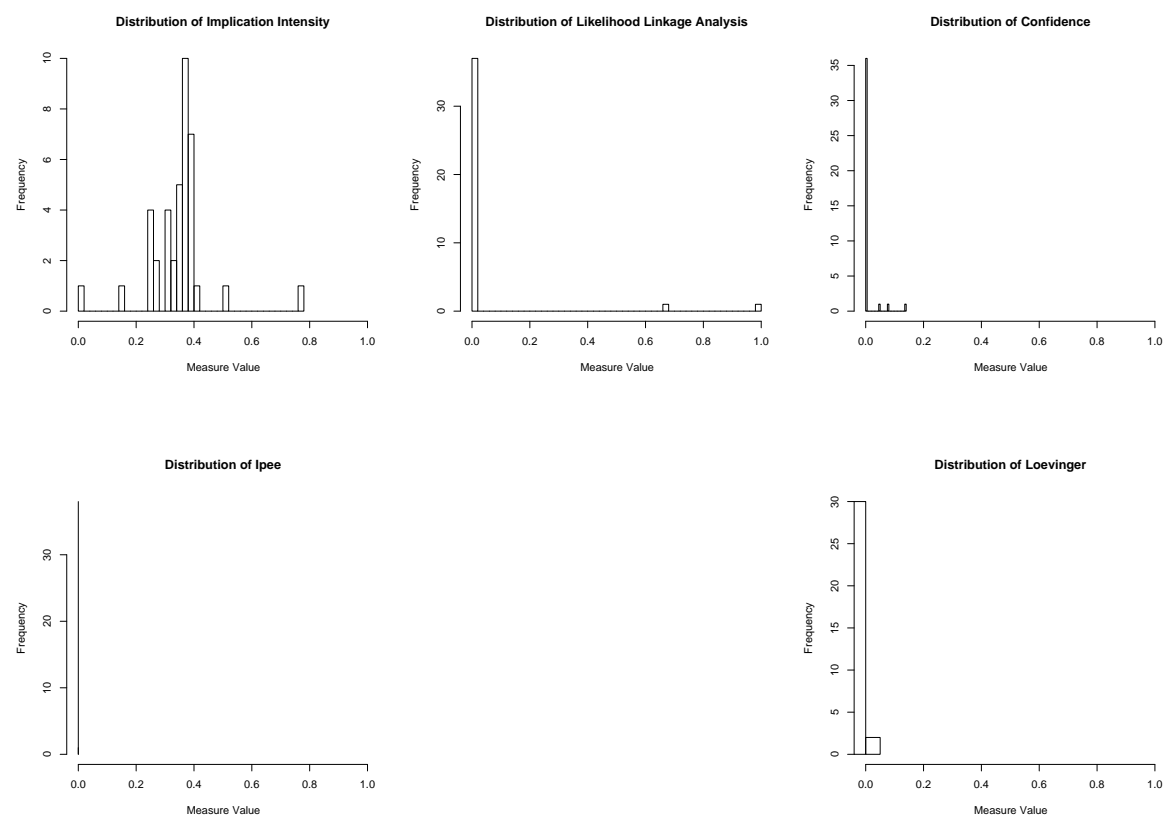

Fig. 6. Measures distributions on irrelevant matching relations $R-$

on this type of rules. We can also notice that the quasi-conjunction measure, LLA, does not distinguish good rules from bad ones.

From these experiments, we conclude that matching relations are better evaluated by IMs of deviation from independence. In such cases, the number of counter-examples needed to reach the equilibrium situation is less than the number needed to reach the independence situation. We also found that the statistic measure of quasi-implication, II, is well suited for distinguish good rules from bad ones.

\section{Conclusion}

In this paper, we proposed an original use of the association rule model and interestingness measures in the context of schema/ontology matching. More precisely, we described the AROMA approach, which is an extensional matcher for hierarchies indexing text documents. A novel feature of AROMA is that it uses of the asymmetrical aspect of association rules in order to discover subsumption matches between hierarchies or ontologies. Based on studies of IMs, we selected several IMs according to three criteria (subject, nature and scope) and we evaluated them on a matching benchmark. The two experiments show 
that deviation from independence measures are the best adapted IM family for such an application since the evaluated rules are good regarding the independence situation, but bad in terms of equilibrium deviation. From these results, we can also argue that the two descriptive indexes used, Confidence and Loevinger, tend to have the same behaviour. Due to its deviation from independence subject, its statistical nature and its quasi-implication scope, the Implication Intensity obtains the best scores on this benchmark. In this paper, we analysed measure behaviour on the process of rule extraction between concepts. We did not study the terminological step of AROMA, which consists of extracting and selecting concept relevant terms. Such an evaluation would be interesting since this terminological extraction process significantly influences the accuracy of the results.

\section{References}

1. R. Agrawal, T. Imielinski, and A. Swami. Mining association rules between sets of items in large databases. In Proceedings of the 1993 ACM SIGMOD International Conference on Management of Data, pages 207-216. ACM Press, 1993.

2. R. Agrawal and R. Srikant. Fast algorithms for mining association rules. In J.B. Bocca, M. Jarke, and C. Zaniolo, editors, Proceedings of the 20th International Conference Very Large Data Bases (VLDB'94), pages 487-499. Morgan Kaufmann, 1994.

3. Jr. Bayardo, J. Roberto, and R. Agrawal. Mining the most interestingness rules. In Proceedings of the 5th ACM SIGKDD International Confeference On Knowledge Discovery and Data Mining (KDD'99), pages 145-154, 1999.

4. J. Blanchard. A visualization system for interactive mining, assessment, and exploration of association rules. PhD thesis, University of Nantes, 2005.

5. J. Blanchard, F. Guillet, H. Briand, and R. Gras. Assessing rule interestingness with a probabilistic measure of deviation from equilibrium. In Proceedings of the 11th international symposium on Applied Stochastic Models and Data Analysis (ASMDA-2005), pages 191-200. ENST, 2005.

6. J. Blanchard, F. Guillet, R. Gras, and H. Briand. Using information-theoretic measures to assess association rule interestingness. In Proceedings of the fifth IEEE international conference on data mining ICDM'05, pages 66-73. IEEE Computer Society, 2005.

7. S. Castano, V. De Antonellis, and S. De Capitani Di Vimercati. Global viewing of heterogeneous data sources. IEEE Transactions on Knowledge and Data Engineering, 13(2):277-297, 2001.

8. S. Castano, A. Ferrara, and S. Montanelli. Matching ontologies in open networked systems: Techniques and applications. Journal on Data Semantics, 3870(V):25-63, 2006.

9. A. Ceglar and J. F. Roddick. Association mining. ACM Computing Surveys, $38(2): 5,2006$.

10. J. Cohen. A coefficient of agreement for nominal scales. Educational and Psychological Measurement, 20(1):37-46, 1960. 
11. J. David, F. Guillet, R. Gras, and H. Briand. Conceptual hierarchies matching : an approach based on discovery of implication rules between concepts. In Proceedings of the 17th European Conference on Artificial Intelligence (ECAI2006), pages pages 357-361, 2006.

12. H.H. Do and E. Rahm. Coma - a system for flexible combination of schema matching approaches. In Proceedings of the 28th International Conference on Very Large Data Bases (VLDB '02), pages 610-621, 2002.

13. A. Doan, J. Madhavan, P. Domingos, and A. Halevy. Learning to map between ontologies on the semantic web. In Proceedings of the 11th International $W W W$ Conference (WWW'02), pages 662-673. ACM Press, 2002.

14. A. Doan, J. Madhavan, P. Domingos, and A. Halevy. Ontology matching: a machine learning approach. In S. Staab and R. Studer, editors, Handbook on Ontologies in Information Systems, pages 397-416. Springer-Velag, 2004.

15. J. Euzenat and P. Valtchev. An integrative proximity measure for ontology alignment. In Proceedings of the Semantic Integration Workshop, 2nd International Semantic Web Conference (ISWC-03), 2003.

16. U. M. Fayyad, G. Piatetsky-Shapiro, P. Smyth, and R. Uthurusamy, editors. Advances in Knowledge Discovery and Data Mining. AAAI/MIT Press, 1996.

17. Alex Alves Freitas. On rule interestingness measures. Knowledge-Based Systems, 12(5-6):309-315, 1999.

18. F. Fürst and F. Trichet. Axiom-based ontology matching. In Proceedings of the 3rd international conference on Knowledge capture (K-CAP '05), pages 195196. ACM Press, 2005.

19. Liqiang Geng and Howard J. Hamilton. Interestingness measures for data mining: A survey. ACM Comput. Surv., 38(3):9, 2006.

20. F. Giunchiglia, P. Shvaiko, and M. Yatskevich. S-match: an algorithm and an implementation of semantic matching. In European Semantic Web Symposium, LNCS 3053, pages 61-75, 2004.

21. R. Gras et al. L'implication statistique, une nouvelle méthode exploratoire de données. La pensée sauvage, 1996.

22. Robert J Hilderman and Howard J Hamilton. Knowledge Discovery and Measures of Interestingness. Kluwer Academic Publishers, 2001.

23. W. Hu, N. Jian, Y. Qu, and Y. Wang. Gmo: A graph matching for ontologies. In Proceedings of the K-CAP 2005 Workshop on Integrating Ontologies, pages 41-48, 2005.

24. R. Ichise, M. Hamasaki, and H. Takeda. Discovering relationships among catalogs. In E. Suzuki and S. Arikawa, editors, Proceedings of the 7th International Conference on Discovery Science (DS'04), volume 3245 of LNCS, pages 371-379. Springer, 2004.

25. R. Ichise, H. Takeda, and S. Honiden. Integrating multiple internet directories by instance-based learning. In G. Gottlob and T. Walsh, editors, Proceedings of the eighteenth International Joint Conference on Artificial Intelligence (IJCAI03), pages 22-30. Morgan Kaufmann, 2003.

26. Y. Kalfoglou and B. Hu. Cms: Crosi mapping system - results of the 2005 ontology alignment contest. In Proceedings of the K-CAP 2005 Workshop on Integrating Ontologies, pages 77-85, 2005.

27. Y. Kalfoglou and M. Schorlemmer. Ontology mapping: the state of the art. Knowledge Engineering Review, 18(1):1-31, 2003. 
28. M. S. Lacher and G. Groh. Facilitating the exchange of explicit knowledge through ontology mappings. In Proceedings of the 14th International Florida Artificial Intelligence Research Society Conference (FLAIRS'01), pages 305309. AAAI Press, 2001.

29. J. Madhavan, P. A. Bernstein, and E. Rahm. Generic schema matching with cupid. In Proceedings of the 27th International Conference on Very Large Data Bases (VLDB'01), pages 49-58, 2001.

30. S. Melnik, H. Garcia-Molina, and E. Rahm. Similarity flooding: A versatile graph matching algorithm and its application to schema matching. In Proceedings of the 18th International Conference on Data Engineering(ICDE'02), pages 117-128. IEEE Computer Society, 2002.

31. H. Nottelmann and U. Straccia. A probabilistic, logic-based framework for automated web directory alignment. In Zongmin Ma, editor, Soft Computing in Ontologies and the Semantic Web, Studies in Fuzziness and Soft Computing, pages 47-77. Springer Verlag, 2006.

32. N. Noy and M. Musen. Anchor-prompt: Using non-local context for semantic matching. In Proceedings of the Workshop on Ontologies and Information Sharing at the 17th International Joint Conference on Artificial Intelligence (IJCAI'01), pages 63-70, 2001.

33. G. Piatetsky-Shapiro. Discovery, analysis and presentation of strong rules. In G. Piatetsky-Shapiro and W. Frawley, editors, Knowledge Discovery in Databases, pages 229-248. AAAI Press/MIT Press, 1991.

34. E. Rahm and P. A. Bernstein. A survey of approaches to automatic schema matching. The VLDB Journal, 10(4):334-350, 2001.

35. P. Shvaiko and J. Euzenat. A survey of schema-based matching approaches. Journal on Data Semantics, 4(LNCS 3730):146-171, 2005.

36. U. Straccia and R. Troncy. omap: Combining classifiers for aligning automatically owl ontologies. In Proceedings of the 6th International Conference on Web Information Systems Engineering (WISE-05), number 3806 in LNCS, pages 133-147. Springer Verlag, 2005.

37. Pang-Ning Tan, Vipin Kumar, and Jaideep Srivastava. Selecting the right objective measure for association analysis. Information Systems, 29(4):293-313, 2004. 